\title{
Design of an Open ICT4D Knowledge Repository
}

\author{
Melanie PLATZ ${ }^{1}$, Judy VAN BILJON ${ }^{2}$ \\ ${ }^{1}$ University of Koblenz-Landau, Fortstr. 7, 76829 Landau, Germany \\ \& University of South Africa, Unisa Science Campus, Florida, Johannesburg, South Africa \\ Tel: +49(0) 634128031 208, Fax: +49(0) 634128031 385, Email: platz@uni-landau.de \\ ${ }^{2}$ University of South Africa, Unisa Science Campus, Florida, Johannesburg, South Africa \\ Tel: +27(0) 11670 9182, Email: Vbiljja@unisa.ac.za
}

\begin{abstract}
Reputable Information and Communication Technology (ICT) for Development (ICT4D) collections focused on Sub-Saharan Africa exist but a platform where these silos of excellence can be aligned and shared for open access is not a reality yet. In this article, we explore questions on how that should be done and propose a first collection of the contents and the functionalities of an open browserbased ICT4D knowledge repository (ICT4DKR). To evaluate and extend this collection, a survey on the usefulness and functionality of a South African ICT4D knowledge repository (SA-ICT4DKR) was performed and will be reported in this article. From the results of the questionnaire, motivators, barriers and constraints on an ICT4DKR were derived.
\end{abstract}

Keywords: ICT4D, Knowledge Repository, Open Source

\section{Introduction}

A core goal of ICT4D is to transform society and create a sustainable environment. The Information Systems discipline has once been described as a fragmented adhocracy that allows various intellectual communities to coexist, (Banville \& Landry, [1]). The same can be seen to apply to the study of ICT in relationship to human and social development (ICT4D). Due to the relative immaturity of the field there is a lack of consensus on some of the fundamental issues such as the research agenda, core theories and frameworks, (Biljon \& Alexander, [2]). The correspondence between the millennium development goals and those of ICT4D has been a major driver of the growing interest in the field of using technology as a means of improving the human condition. While that is still an important set of indices there is some concern that it harbours an externally controlled agenda, (Chaudhuri, [3]), and therefore recent ICT4D thinkers emphasise the locally set agenda and a less quantitative approach. At the end of 2015, the Millennium Development Goals will be replaced by the post-2015 development agenda. Heeks, [4], identified a set of post-2015 priorities in international development which have to date been under-emphasised within development informatics. Nikolidakis, [5], discusses critical success factors for development projects dealing with ICT and emphasises that good cooperation and a functioning network of the project's stakeholders are vital to the success of an ICT4D project. Furthermore, Nikolidakis, [5], identifies sharing goals or "the same dream" among the organisations involved as very important. This again raises the need to communicate and share ideas and resources. To improve communication and ensure a solid foundation for future research sharing of the basic objectives, assumptions, central theories, models and frameworks of ICT4D is necessary. The University of South Africa (UNISA), which has many post-graduate students living and working in different parts of South Africa and Africa is in a unique position to develop a network of research collaborators. Therefore, 
UNISA is proposed as the host for the development of an open knowledge repository where research agendas, methodologies, theories, models and frameworks are investigated and discussed for inclusion in the open ICT4DKR. The goal is to create a platform where the already reputable ICT4D collections can be aligned and shared for open access. This raises question on how it should be approached in order to meet the criteria of an open but organised and maintained knowledge repository and that is the rationale for this study.

\section{Methodology}

\subsection{Objectives}

The objective of this study is to explore the following question: How can an inclusive ICT4D knowledge repository be developed? To reach this goal, architecture for the comprehensive inclusion of different ICT4D research groups in an ICT4DKR has to be developed. The current scope is South Africa but the idea is to extend this for sub-Saharan Africa. Therefore, a generative method has to be developed. The architecture for the knowledge repository should support collaboration between ICT4D research groups, practitioners and communities. Sub-groups working on the different aspects of the knowledge repository as informed by the architecture have to be created and the contributions of the sub-groups have to be evaluated constantly. Awareness of potential usefulness of the knowledge repository has to be raised, e.g. via presentations on conferences or articles in popular journals. To optimise the knowledge repository, knowledge on African tradition and culture should be captured continuously as this knowledge relates to ICT development.

\subsection{Strategy}

The initial architecture was compiled from literature and refined by considering similar knowledge repositories. A first collection of contents and the functionalities of an open browser-based ICT4DKR was thus compiled. To evaluate and extend this collection, a survey on the usefulness and functionality of an SA-ICT4DKR was conducted. The results of the survey will be analysed to derive the requirements and constraints for an open $S A$ ICT4DKR.

\section{Literature overview}

\subsection{Knowledge Repository Architecture}

"A repository is an institutional-scale collection which feeds off individual's document collections.”, Mosweunyane \& Carr, [14], p. 1. Knowledge Repositories for different fields have been developed, e.g. for e-Infrastructure (cf. Castelli, Taylor \& Zoppi, [6]), or eAgriculture (cf. Lwoga, Forzi, Laing \& Mjema, [7]). A socio-technical analysis is needed to identify the key aspects that impact the design, uptake and usage of a shared knowledge repository. Therefore, we proceed in a similar way like Lwoga et al., [7], who used an exploratory survey, system analysis and ICT-based design as methodologies to convey the current state of knowledge sharing activities and assess the user requirements. Towards understanding key aspects that impact the design, uptake and usage of a shared knowledge repository in an information portal Foth, Gonzalez and Kraemer, [8], proposed a model consisting of three layers: a technological layer, a social layer, as well as a discursive layer. Considering the technological layer, the knowledge repository should be freely accessible via several digital devices, e.g. smartphones, tablets, PCs or Digital Doorways. For the repository, the import and export of data (i.e. knowledge) in several data formats should be enabled (cf. section 4). Additionally, a possibility to use the open ICT4DKR offline should 
be implemented, as internet connection is not available everywhere. In the online-version, a platform for discussions should be integrated (cf. section 4). The knowledge repository envisaged is different from an information portal since the latter has no offline communication but the issues of local and global contexts as well as those of interaction paradigms need to be considered. Foth et al., [8], note the importance of the portal design to allow for interoperability with other communication technologies. "If the portal is able to add value to the existing portfolio of devices and services residents employ, it has a chance of becoming an attractive addition or enhancement to the existing communicative ecology.” (Foth et al., [8], p. 40). Considering the social layer, the goal is to develop an open ICT4DKR. Therefore an analysis of the socio-economic-, cultural- and ICT-situation of the target group needs to be done as well as a software analysis. Considering the discursive layer, the portal designs have to fulfil different needs for different users at different times. Foth et al., [8], sees variability and diversity as crucial advantages and introduced communicative ecology as conceptual response to the challenge of taking variability and diversity into account. In order to face the challenge of taking variability and diversity into account in our case and in order to reach as many potential users as possible and to enable the collaboration of several different user groups, an adaptive Graphical User Interface (GUI) is proposed for the open ICT4DKR.

\subsection{Knowledge Repository Developments}

Current developments in the knowledge repository domain were investigated. Castelli et al, [6], describe the development of a platform for e-Infrastructure on order to fill the gap separating the e-Infrastructure providers from the users. This platform enables eInfrastructure providers and users to collaborate and exchange knowledge, which ensures that e-Infrastructures are developed and effectively used worldwide. This contributes to the emergence of a competitive knowledge-based economy within the project BELIEF. Castelli et al., [6], created a “one-stop-shop" providing a portal and a digital library with a huge number of e-Infrastructures open access publications. The digital library offers continuously updated information on e-Infrastructures-related projects, initiatives and events and provides services to support the submission, description, searching, browsing, retrieval, access, preservation and visualisation of multimedia documents. Castelli et al., [6], emphasise that the technology adopted by BELIEF can be easily adapted to meet the information and collaborative needs of other scientific communities. Lwonga et al., [7], describe a prototype, Agrinet-Tz, which strives to promote development and knowledge sharing among the agricultural researchers in Africa. Its focus is on the application of both the open access and the community of practice as knowledge management approaches, in order to enhance the capturing and sharing of the agricultural knowledge to researchers, farmers and other stakeholders for the sustainable agricultural development in Africa. Orlic, Maliqi \& Misley, [12], give an overview of the web based video educational portal Videolectures.Net, an online platform for promoting education, information and knowledge based technologies, their usability, accessibility, interactive implementation and the ability of sharing. The Labour Market Intelligence Partnership (LMIP), (Reddy, [13]), is a collaboration between government and a national research consortium that aims to build a credible institutional mechanism for skills development in South Africa. Additionally, ISTAfrica (http://www.ist-africa.org/) maintains a currently updated portal containing information and resources facilitating and supporting development of the information society and knowledge economy in Africa. In a similar manner, GESCI (http://www.gesci.org/) provides services and products that create value for stakeholders in the field of ICT for education and development. The ICSU Regional Office for Africa (ICSU ROA) (http://www.icsu.org/africa) sees its role, among others, in collecting and circulating information among scientists and facilitate free flow of scientists and scientific 
knowledge across borders, to establish a database of African experts in all fields of science, to encourage and improve capacity building in Africa and to promote indigenous \& traditional knowledge \& skills. Another knowledge repository is hosted by the Association for the Development of Education in Africa (ADEA) (http://www.adeanet.org/portalv2/en) as forum for policy dialogue which is, among others, a network of policy-makers, educators and researchers, and, based on its capacity to foster policy dialogue and pool ideas, experience, lessons learned and knowledge, a catalyst for educational reform. It is recognised today as being a major actor in the processes of dialogue, sharing and learning for qualitative change in education aimed at promoting Africa's development. Furthermore, the United Nations Economic Commission for Africa (ECA) Library (https://libcat.uneca.org/) is to facilitate the commission to serve as an integrated information service and resource centre for Africa. Among others, the library includes an EResources Collection. Another example is the African Virtual University (http://www.avu.org/).

\section{Proposed ICT4D Repository}

A Content-Management-System (CMS) is considered feasible to fulfil the mentioned requirements and constraints. In the repository, documents providing information and data on ICT4D shall be collected. Everybody is allowed to contribute, but the submitted contributions are not published immediately, but reviewed by experts whose approval is required for publication. Thus, the wiki-idea is combined with a reviewing-process. Contributions on the topics E-Government, E-Health and E-Learning shall be present in the repository. Initially the following publication types shall be available in the repository: books, papers, theses, reports, presentations and articles of junior researchers. That is, junior researchers can upload articles that have not been published yet. By doing this, those articles can be made available to the researcher-community and the contributions can be discussed and remarks or suggestions for improvement can be provided to the author. The aim is to help junior researchers to develop good quality papers, which may pass a reviewing-process for journals or conferences. This idea is based on the concept of arXiv. arXiv.org is a highly-automated electronic archive and distribution server for research articles. Covered areas include physics, mathematics, computer science, nonlinear sciences, quantitative biology and statistics. arXiv is maintained and operated by the Cornell University Library with guidance from the arXiv Scientific Advisory Board and the arXiv Sustainability Advisory Group, and with the help of numerous subject moderators. Users can retrieve papers from arXiv via the web interface. Registered authors may use the web interface to submit their articles to arXiv. Authors can also update their submissions if they choose, though previous versions remain available. (cf. Cornell University Library, [15]). As mentioned, an adaptive GUI is proposed since a GUI on a digital device allows tailored information to different user groups to be provided. "The primary motivation for adapting user interfaces is to improve users’ performance and satisfaction.”, Gajos, Czerwinski, Tan \& Weld, [9], p. 2 . Adaptivity can be defined as follows: A system is adaptive when the user model is altered during interaction with the user, to reflect a changed view of her/him, (Greenberg \& Witten, [10]). A generative mathematical structure for a system adapting a GUI to a user was developed in Platz, [11]. The system responds to the user with automatic adaptivity and suggestions for adaption, which is similar to the method of behavioural targeting. To enable the system to provide an appropriate GUI and thus appropriate decision support, situative analysis has to be included. In order to adapt the GUI to South African conditions it is important to determine an initial state for the GUI. Using an empirical pilot study carried out in Germany, a generic basal concept for the structure of further empirical studies to be performed in South Africa was developed in Platz, [11]. Certain GUI elements are supposed to be evaluated by empirical studies carried out in South Africa with regard to 
adaptivity, comprehensibility, conceiving the spatial sense, benefit for the user and creation of a knowledge repository, among others. Consequently, Concerto, an open source (OS) online $R$-based adaptive testing platform, would eventually be a suitable tool for the implementation of an open ICT4DKR with an adaptive GUI. The collection of contents and the functionalities of an open browser-based ICT4DKR are inspired by these current developments in the domain of knowledge repositories. Consequently, it includes the topics "Repository”, “News”, “Events”, "Resources” and "Personal Profiles”, see Figure 1.

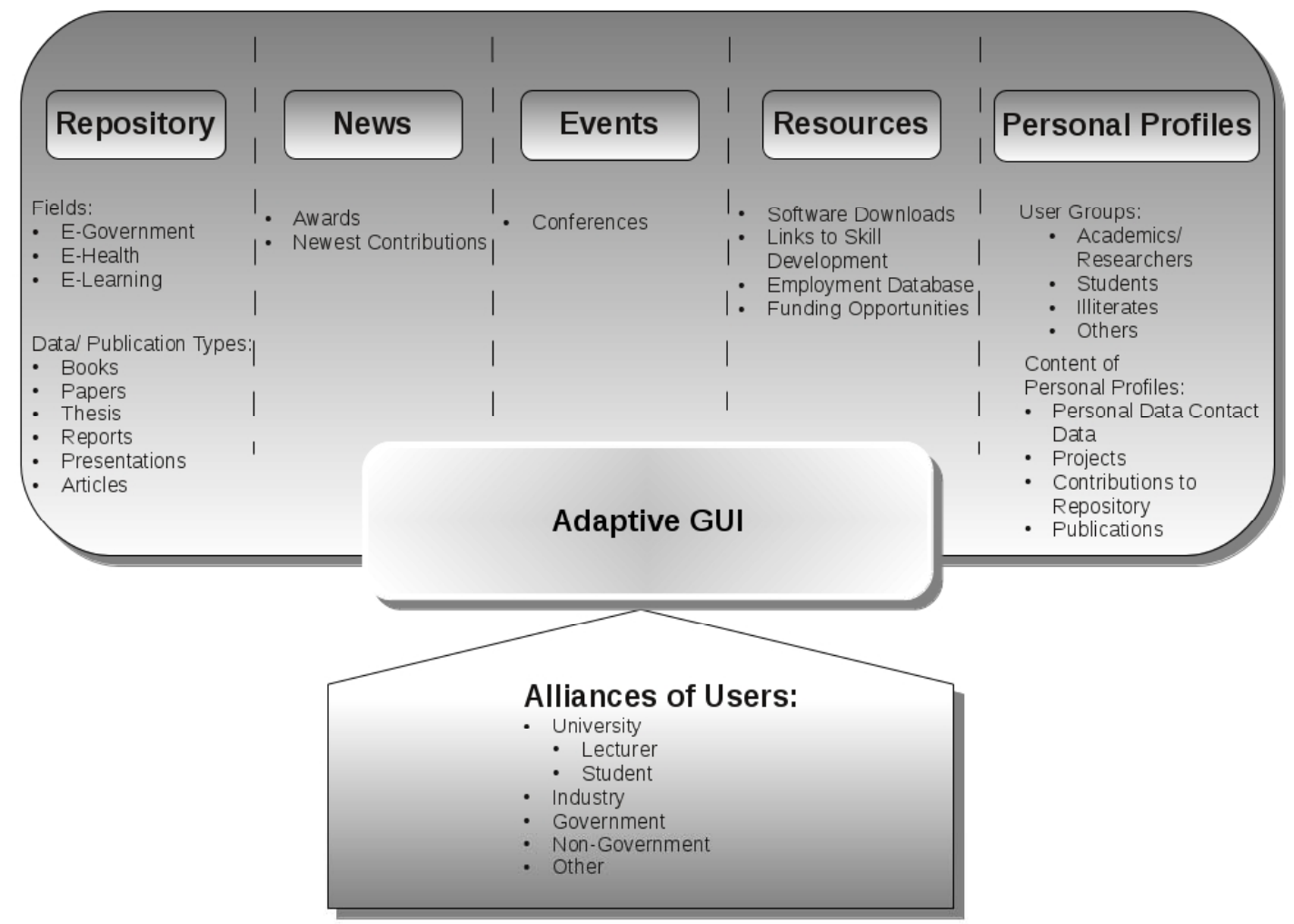

Figure 1: First Draft of the ICT4D Knowledge Repository

\subsection{News}

"News" consists of the subtopics "Newest Contributions" and "Awards". For users of the repository the possibility to evaluate or rate contributions should be implemented. The best evaluated contribution of a certain time-period should be awarded. By doing so, contributors shall be acknowledged and an incentive to contribute to the knowledge repository shall be provided.

\subsection{Events}

Events like conferences or workshops shall be advertised organised on the ICT4DKR. The total cost of ownership (TCO) for participants in such a conference should be kept as low as possible. Therefore, we follow the concept of Niehaus, [16]: The basic principle of reducing the TCO for members implies that more communities with financial constraints can apply software and content. The reduction of the financial threshold to participate in a conference was significantly reduced by the method of Niehaus, [16], which ensures a virtual participation without the necessity of physical attendance. The participants can take part via Virtual Participation Mode either from their place of work in their member states or from regional organised meeting points in the different member states. The communication platform of the conference is a browser-based videoconference software, for example Flashmeeting or OpenMeeting. 


\subsection{Resources}

Under the topic "Resources" software downloads could be made available for the user. The following are examples of OS or free software:

- Text processing: LibreOffice, a powerful office-suite,

- Calculations: $R$, a free software environment for statistical computing and graphics,

- Data collection: OpenDataKit, a set of tools which help organisations author, field, and manage mobile data collection solutions, LimeSurvey, an online survey application, $S D A P S$, an optical mark recognition program which has an integrated workflow with both LibreOffice and LaTeX to create questionnaires.

Additionally, links to skill development, e.g. tutorials for the creation of a good article or thesis or links to science and research networks, e.g. ResearchGate or LinkedIn, employment databases e.g. in Germany Academics, funding opportunities, e.g. in Germany $D F G, B M B F$ or $D A A D$ could be provided.

\subsection{Personal Profiles}

For users of the knowledge repository the possibility to create an own user profile shall be implemented. Such a personal profile should contain personal data, contact data, projects, contributions to the repository and publications. Those user profiles are moderated and checked by an administrator.

\subsection{User Groups}

Researchers like students, academics and lecturers can present themselves in order to enlarge their possibilities to find funders or to receive job offers. They can discuss issues (e.g. research approaches) and learn from others. They can network with other users and create open knowledge by contributing to the knowledge repository. Employers or funders like governmental or non-governmental facility members and industry workers can benefit from the knowledge repository by recruiting suitable employees or project partners. The open browser-based ICT4DKR will first be available in English language. In further work, the knowledge repository could be enhanced to be usable by a larger target group, that is other ICT4D stakeholders beyond the research community. Therefore, the content will be translated to other languages and the icons will be adapted to the cultural background of the user group, if necessary. To enable illiterate users to access the open browser-based ICT4DKR, an icon-based interface will be developed. Video-tutorials, e.g. on how to use ICT, could provide access and meaningful information to support illiterate users.

\section{Evaluation Survey}

A questionnaire on the potential usefulness and desired functionality of an open SAICT4DKR was performed with Limesurvey. This questionnaire is available at the following link: http://mathematik.uni-landau.de/download/Platz/Questionnaire.pdf

The survey was supposed to emphasise the significance of an open SA-ICT4DKR for the probands (respondents) and the opinion of the probands about the usefulness and functionality of an open SA-ICT4DKR. The questionnaire comprises approximately ten questions covering the categories affiliation and position of the proband, functionalities required and some general open ended questions on the implementation of an open $S A$ ICT4DKR.

\section{Results}

The survey had 25 probands participate in completing the online questionnaire. 


\subsection{Affiliation and Position}

Persons from the UNISA (59.09\%), University of Cape Town (UCT) (13.64\%), University of Pretoria (UP) (9.09\%) and Wits University (4.55\%) participated in the questionnaire, see Figure 2.

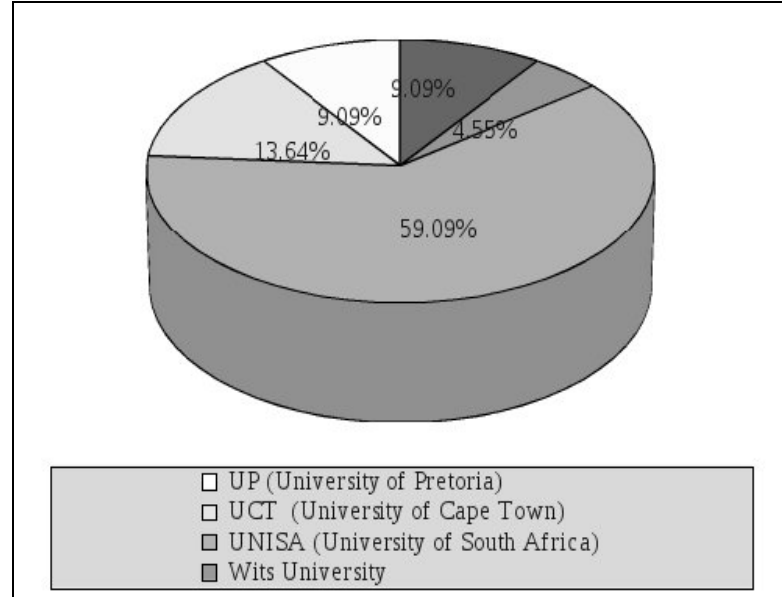

Figure 2: Geographical dispersion

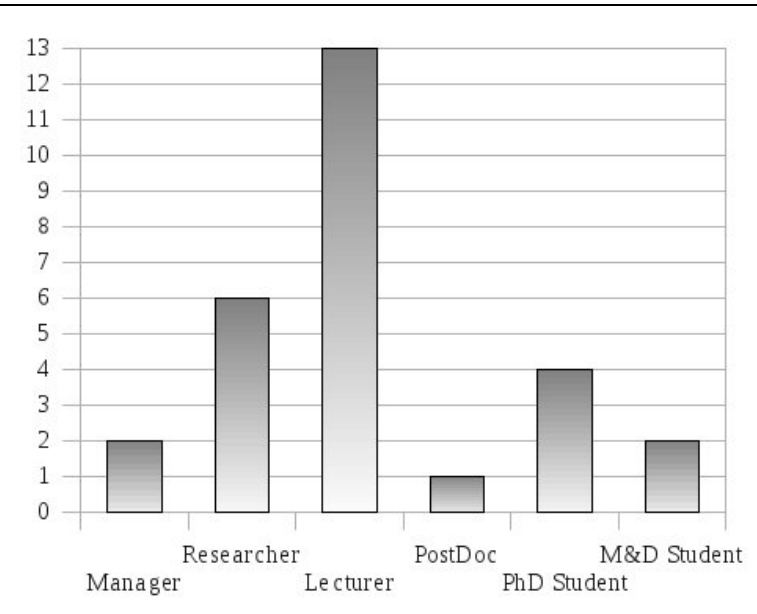

Figure 3: Employment position

Most of the participants have a position as lecturer (see Figure 3). Researchers and PhD Students are the second largest group. Furthermore, managers, M\&D Students and PostDocs also participated.

\subsection{Functionalities/Contents of the Repository}

Fuzzy logic is used to represent uncertainties and ambiguities in linguistic descriptions. It allows representing properties with a value of truth in the range between 0 and 1 . In Figure 4 the mean value of the degree of importance of different functionalities and contents of the repository is visualised. The degree of importance is a real number in the interval from 0 to 1 with "0" for "not important at all" and " 1 " for "very important". Most important to the probands are the Resources: Links to research outputs from SA authors (degree of importance: 0.52). Furthermore, the following items are evaluated as important (i.e. degree of importance larger than or equal 0.5): Links to journal articles (degree of importance: 0.5) and Resources: Dissertations ( $M \& D$ ) (degree of importance: 0.5 ). The following items can be indicated as less important (degree of importance lower than 0.4): Resources: Software downloads (degree of importance: 0.39), Adaptive graphical user interface tailored to user groups, e.g. industry, students, researchers (degree of importance: 0.39), Resources: Employment databases (degree of importance: 0.39) and ICT4D career news and vacancies (degree of importance: 0.37). One proband points out that "a balance should be struck between carrying useful information and not flooding people's mail boxes" and another proband mentions that search facilities by year, author, keywords, etc. should be implemented. 


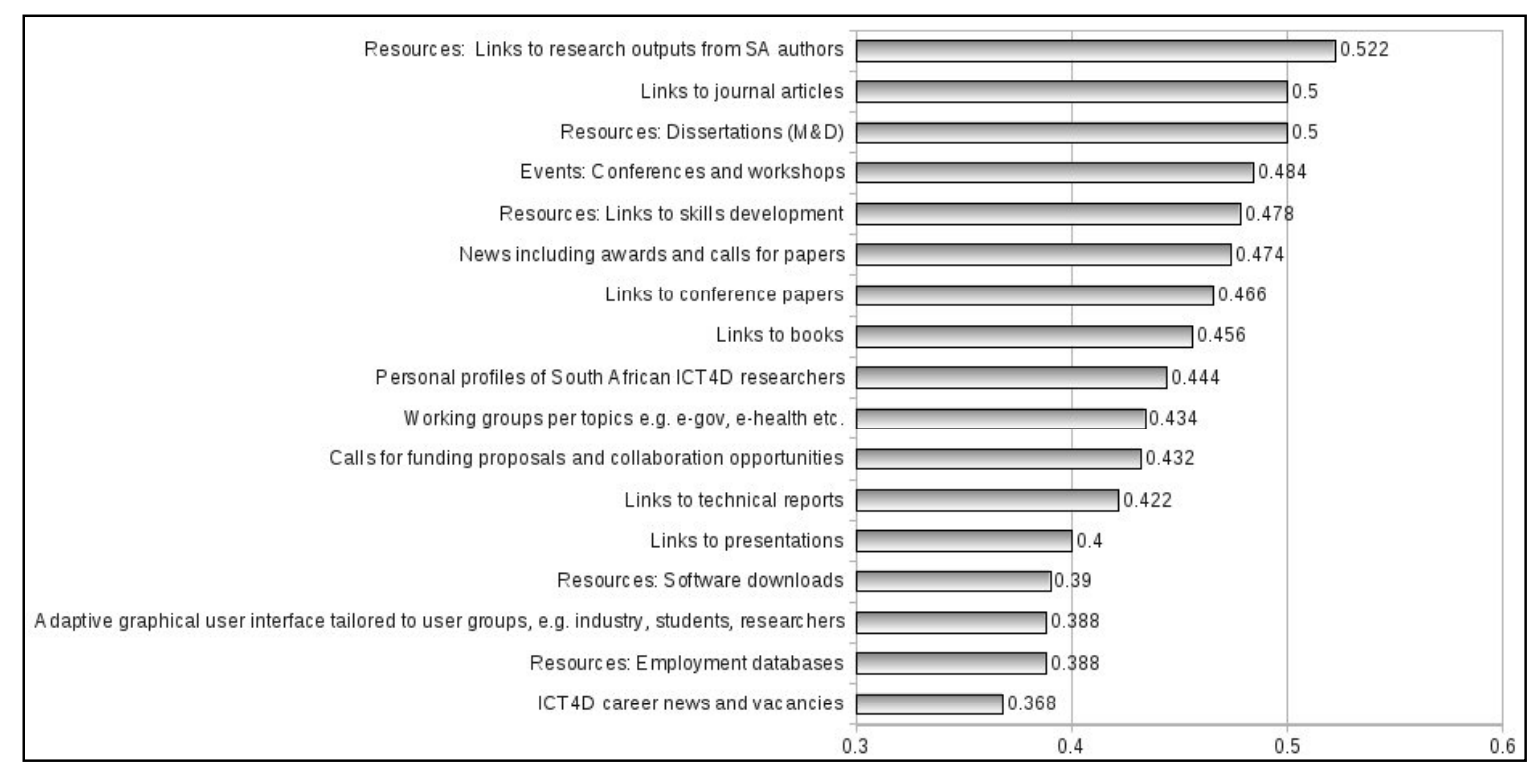

Figure 4: Evaluation of the Functionalities/ Contents of the Repository (Mean Value).

\subsection{Is There a Need for a SA-ICT4DKR?}

The majority of probands think, that there clearly is a need for an SA-ICT4DKR. One proband states, that South Africa should be driving research in ICT4D as this will bring together a fragmented community. Another proband adds: "I think there are many particularities on the South African context (historical, social and cultural) that make it unique, and therefore necessary to reflect upon research done in the same circumstances." Yet another proband indicates, that an interest in something like this was clearly expressed at the South African Institute of Computer Scientists and Information Technologists Conference SAICSIT 2014. But the proband cautions that people are sometimes eager in principle but not participating in practice. Other probands indicate, that an SA-ICT4DKR would be useful to have an easy way of finding out who is working in the area and what they are doing to avoid conflict and to have the opportunity to get in touch with other researchers when necessary. An SA-ICT4DKR enables easier access to relevant sources for research in a specific field. It will strengthen the quality of work, reduce overlap, increase synergy and avoid previously encountered pitfalls. Furthermore, it is assumed, that an $S A$ ICT4DKR will make it easier to attract $M \& D$ students in the project. One proband concludes: "Knowledge is a powerful resource that enables individuals and organizations to achieve several benefits such as improved learning and decision-making. Having a South African Knowledge repository will help with those achievements.”

\subsection{Motivators for a Shared Knowledge Repository}

The following motivators are indicated by the probands: A single unified portal with content by (South) Africans and specific to South Africa and Africa in general is useful to create awareness of what research is going on in ICT4D in (South) Africa, that is, e.g. what workshops and conferences are organised. Furthermore, duplication and "re-inventing the wheel” can be avoided through the use of a shared knowledge repository by reducing an overlap between projects. Additionally, coordination, collaboration, knowledge sharing and "learning from others" can be performed via a shared knowledge repository. Thereby, a critical mass or cohesive group can be created and the international impact of research results can be increased. Furthermore, the momentum in the project could be enhanced and new researchers could be attracted. One proband states: "The only other one is in the UK. It is embarrassing that there is none in Africa - in the midst of where we have the greatest 
need.”

\subsection{What are the Barriers to a Shared Knowledge Repository?}

One barrier seen by the probands is a potential lack of "buy in" from participants, i.e. sometimes a shared space is created and not used, and basically the lack of check and balances to ensure that the repository is regularly updated. Another barrier indicated is time and effort to get it in place. Furthermore, one proband points out, that patents and intellectual property clauses restrict the open use of knowledge, additionally the "workingin-silo mentality" could be obstructive. Another consideration is, that "limited overall funding e.g. from National Research Foundation (NRF) may hold some researchers back from sharing fully." Some probands apprehend that it could get too big, while others apprehend that there could be a lack/shortage of researchers contributing to the knowledge repository. Another mentioned barrier could be poor design to back- and front-end facilities. Nevertheless, the comment "I still think it is worth trying" is formulated by one proband.

\subsection{Would it be Feasible to Host the Knowledge Repository at a University?}

Most of the probands have the opinion that it is feasible to host the knowledge repository at a university, especially if there is funding so that the repository could be well managed. One proband gives the following example: "Indeed it would be. We currently have other knowledge repositories hosted. For instance, the NETD portal (http://www.netd.ac.za) is hosted by NRF but maintained by UCT." Yet another proband emphasises: "Yes, particularly at UNISA - UNISA is "non-aligned"." Other probands see a possibility to host the knowledge repository at a university, if there is a champion who has access to the resources to host it. Some probands have the opinion that the knowledge repository should ideally not be hosted at a university, but on an "independent" URL or host or as cloud based application. The CMS could then be managed by a university. The proband sees a possibility to host the repository at a university as long as it is clear that this is done for logistics reasons, the content is not "owned" by the university and the repository can be copied/moved to another institution if members wish so. Another proband states that continuity issues may arise if the key researchers driving the issue at the university leave or retire. Then it will be quite difficult to move the resource to another "hosting institution".

\subsection{Comments and Suggestions}

One proband states, that South Africa should be driving research in ICT4D as this will bring together a fragmented community. One proband asks for tentative timelines with regards to when this repository might be set up. Another proband suggests to start a Google site with invited contributors as a way of starting the repository. And other probands state: "Great idea. Keen to be part of the initiative."; "This is an impressive project. I will be happy if it can be done well taking into account the best practices from the UK one (Richard Heeks) and adding what is really unique about ICT4D projects.”

\section{Business Benefits}

The concept of Open Community is used as measure and draft strategy for a participative communication and an efficient knowledge management. Open Community is a generalisation of the concept of OS to other collaborative effort. The term "open" for an open community refers to the opportunity for anyone to join and contribute to the collaborative effort. The direction and goals are determined collaboratively by all members of the community. The resulting work ("product") is made available under a free license, so that other communities can adapt and build on them, Niehaus, [17]. Using this concept, 
already developed components of OS software and Open Content can be modified and adjusted for the development of an application. The advantage is faster development, improvement and distribution of the application. The application can be provided free of charge.

\section{Future Work}

In future work, a Product Requirements Document (PRD) should be developed based on the requirements and constraints of the open ICT4DKR. From this PRD the requirements to the software tools and hardware tools can be derived and a software analysis to identify already existing OS software that can be used directly or in a modified way for the creation of the open ICT4DKR can be performed. An open browser-based prototype for an open browserbased ICT4DKR should be created based on the collection of contents and the functionalities of an open ICT4DKR and the results of the questionnaire. Further surveys on the quality of the prototype of the open ICT4DKR should be performed. Based on the results, the prototype should be optimised and an improved version should be tested in an empirical study with pre-test, post-test and follow-up-test.

\section{Conclusions}

In this article, a first collection of the contents and the functionalities of an open browserbased ICT4DKR were proposed. To extend this collection, the proposal of contents and functionalities was evaluated with a survey on the potential usefulness and desired functionality of a SA-ICT4DKR. The need for an open SA-ICT4DKR is confirmed by the majority of $84.21 \%$ of the probands (15.79\% abstentions). The evaluation of the contents and the functionalities proposed for an open browser-based ICT4DKR provided useful insights into the motivators, barriers and constraints. The number of 25 participants in the survey is low, therefore another survey aiming at a larger target group should be performed. The initial prototype is designed for the South African research community. Transferability cannot be assumed therefore evaluation and related customisation would be required to make the contents meaningful to the wider Sub-Saharan community. By taking the development of an open ICT4DKR further, the ideal of a platform where reputable ICT4D collections focused on Sub-Saharan Africa can be aligned and shared, can become a reality.

\section{References}

[1] Banville, C. \& Landry, M. (1989). Can the Field of MIS be Disciplined? Communications of the ACM 32.1: 48-60.

[2] Biljon, J. \& Alexander, T. (2014). Information and Communication Technology for Development (ICT4D) research: the quest for a shared conceptual framework continues. Paper presented at the 8th International Development Informatics Conference (IDIA2014), Port Elizabeth, South Africa.

[3] Chaudhuri, A. (2012). ICT for Development: solutions seeking problems? Journal of Information Technology, 27(4), 326-338.

[4] Heeks, R. (2014). Development Informatics 1-44. Retrieved 13 June 2014 from. http://www.seed.manchester.ac.uk/subjects/idpm/research/publications/wp/di/

[5] Nikolidakis, N. (2007). It's Not (Only) About Technology - Success Factors in ICT4D-Projects. ISTAfrica Conference, 2007, Mozambique, IIMC International Information Management Corporation.

[6] Castelli, D., Taylor, S.J.R. \& Zoppi, F. (2010). Open knowledge on e-Infrastructures: the BELIEF project Digital Library. IST-Africa, 2010 , vol., no., pp.1,15, 19-21 May 2010.

[7] Lwoga, E., Forzi, T., Laing, P. \& Mjema, E. (2006). KM in the agricultural field: an ICT-based approach to promote the development and sharing of knowledge among agricultural researchers in Africa. ISTAFRICA Confererence.

[8] Foth, M., Gonzalez, V. M. \& Kraemer, K. L. (2008). Design Considerations for Community Portals in Master-Planned Developments in Australia and Mexico. Paper presented at the OZCHI , December 8-12, , Cairns, QLD, Australia.

[9] Gajos, K. Z., Czerwinski, M., Tan, D. S. \& Weld, D. S. (2006, May). Exploring the design space for adaptive graphical user interfaces. In Proceedings of the working conference on Advanced visual 
interfaces (pp. 201-208). ACM.

[10] Greenberg, S. \& Witten, I. H. (1985). Adaptive personalized interfaces-A question of viability. Behaviour \& Information Technology, 4(1), 31-45.

[11] Platz, M. (2014). Mathematical Modelling of GIS Tailored GUI Design with the Application of Spatial Fuzzy Logic. Universität Koblenz-Landau. http://kola.opus.hbz-nrw.de/volltexte/2014/965/

[12] Orlic, D., Maliqi, L. \& Misley, S. (2008). Online Video-educational Repository for Knowledge based Training, Innovation and Scientific Research - Case Study. IST-Africa Conference, 2008, IIMC International Information Management Corporation.

[13] Reddy, V., Labour Market Intelligence Partnership. http://www.lmip.org.za/, retrieved: 16/12/2014.

[14] Mosweunyane, G., \& Carr, L. A. (2014, May). Direct desktop-repository deposits with SWORD. In ISTAfrica Conference Proceedings, 2014 (pp. 1-8). IEEE.

[15] Cornell University Library. ArXiv.org.http://arxiv.org/, retrieved: 16/12/2014.

[16] Niehaus, E. (2013). AT6FUI - Agenda Workshop 2013.

http://at6fui.weebly.com/agenda-workshop-2013.html, retrieved: 16/12/2014.

[17] Niehaus, E., Definition: Open Community - Action Team 6 Follow-Up Initiative. http://at6fui.weebly.com/open-community-approach.html, retrieved:16/12/2014. 